\title{
PROPER HOLOMORPHIC MAPPINGS EXTEND SMOOTHLY TO THE BOUNDARY
}

\author{
BY STEVEN BELL AND DAVID CATLIN
}

Biholomorphic mappings between smooth bounded domains in $\mathbf{C}^{n}$ are known to extend smoothly to the boundary in a wide variety of cases $[7,5,1]$. Much less is known about the boundary behavior of proper holomorphic mappings. In this communication, we sketch the proof of

THEOREM 1. Suppose $f: D_{1} \rightarrow D_{2}$ is a proper holomorphic mapping between smooth bounded pseudoconvex domains contained in $\mathbf{C}^{n}$. If the Bergman projection associated to $D_{1}$ maps $C^{\infty}\left(\bar{D}_{1}\right)$ into $C^{\infty}\left(\bar{D}_{1}\right)$, then $f$ extends smoothly to $\bar{D}_{1}$.

Kohn has proved that the Bergman projection associated to a smooth bounded domain $D$ maps $C^{\infty}(\bar{D})$ into $C^{\infty}(\bar{D})$ when $D$ is strictly pseudoconvex [11], and more generally, when the boundary of $D$ satisfies certain geometric conditions [12]. Diederich and Fornaess [6] have shown that these conditions are satisfied when the boundary of $D$ is real analytic and pseudoconvex.

REMARKs. (A) Our proof of Theorem 1 uses arguments similar to those used in [2] where it was assumed that the Bergman projection preserved the space of functions which are real analytic up to the boundary. The additional complications encountered in the present work stem from the fact that the ring of germs of smooth functions is not a unique factorization domain.

(B) K. Diederich and J. E. Fornaess have informed us that they also have obtained a proof of Theorem 1 [8].

SKetch of THE PROOF OF TheOREM 1. A complete proof of this theorem will appear in [4]. In [3], it is shown that under the hypotheses of Theorem 1, the Jacobian determinant of $f, u=\operatorname{Det}\left[f^{\prime}\right]$, extends smoothly to $\bar{D}_{1}$ and $u f^{\alpha}$ extends smoothly to $\bar{D}_{1}$ for each multi-index $\alpha$. Hence, we are faced with a division problem: to show that $u$ divides $u f$ in $C^{\infty}\left(\bar{D}_{1}\right)$, given that $u$ and $u f^{\alpha}$ are in $C^{\infty}\left(\bar{D}_{1}\right)$ for each $\alpha$. A necessary first step in attempting to solve this division problem is

LEMma 1. Under the hypotheses of Theorem 1 , the Jacobian $u=\operatorname{Det}\left[f^{\prime}\right]$ vanishes to at most finite order at each boundary point of $D_{1}$.

Received by the editors January 6, 1982.

1980 Mathematics Subject Classification. Primary 32H99; Secondary 32 A40.

() 1982 American Mathematical Society 0273-0979/82/0000-0297/\$02.00 
We shall describe the proof of Lemma 1 at the end of this note.

The main tool used to prove Theorem 1 is an adapted version of the Mather division theorem. Let $U$ be the upper half plane $\{\operatorname{Im} z>0\}$ in $\mathbf{C}$, and let $\bar{U}$ be its closure. Let $\Gamma^{N}$ denote the set of functions $h(z, x)$ which are defined in a neighborhood of $(0,0)$ in $\bar{U} \times \mathbf{R}^{N}$, which for fixed $x$ are holomorphic in $z$ on $U$, and which are smooth up to the boundary of $U \times \mathbf{R}^{N}$ near $(0,0)$. Suppose that $F(z, x)$ and $G(z, x)$ are in $\Gamma^{N}$ and that $\left(\partial^{m} F / \partial z^{m}\right)(0,0) \neq 0$. Then there exist a neighborhood $W$ of the origin in $\mathbf{C} \times \mathbf{R}^{N}$, a function $Q(z, x)$ in $\Gamma^{N}$, and smooth functions $a_{k}(x)$ such that for $(z, x)$ in $W \cap \bar{U} \times \mathbf{R}^{N}$,

$$
G(z, x)=Q(z, x) F(z, x)+\sum_{k=0}^{m-1} a_{k}(x) z^{k}
$$

The proof of this division theorem is a straightforward modification of the proof of the Mather division theorem given in, for example, [10]. In the usual way, an analogous version of the Malgrange preparation theorem follows for the class $\Gamma^{N}$.

We now make a change of variables to place the functions $u$ and $u f^{\alpha}$ in the space $\Gamma^{2 n-2}$. Let $z_{0}$ be a point in the boundary of $D_{1}$ such that $u\left(z_{0}\right)=0$, and let $\left(z_{1}, z_{2}, \ldots, z_{n}\right)$ be holomorphic coordinates in a neighborhood of $z_{0}$ such that

(i) the $z_{1}$ direction is transverse to the boundary of $D_{1}$ at $z_{0}$, and

(ii) $u$ vanishes to finite order $m$ at $z_{0}$ in the $z_{1}$ direction.

Let $x \in \mathbf{R}^{2 n-2}$ be given by $x=\left(x_{2}, y_{2}, \ldots, x_{n}, y_{n}\right)$ where $z_{k}=x_{k}+i y_{k}$. For fixed $x$, we may apply the Riemann mapping theorem in the variable $z_{1}$ to flatten out the boundary. Furthermore, this can be done smoothly in $x$. Under this change of variables, a holomorphic function on $D_{1}$ in $C^{\infty}\left(\bar{D}_{1}\right)$ is transformed into a function in the class $\Gamma^{2 n-2}$.

Let $f_{1}$ denote the first component of the mapping $f$. The functions $u$ and $u f_{1}^{j}$ are in $\Gamma^{2 n-2}$ for $j=1,2,3, \ldots$ We now apply the division theorem to $G=u f_{1}$ and $F=u$. We obtain

$$
u f_{1}=q u+r
$$

where $r$ is a polynomial in $z=z_{1}$ of order $m-1$ with smooth coefficients in $x$. Note that $f_{1}=q+r / u$. A simple induction argument using the fact that $u f_{1}^{j}$ is in $\Gamma^{2 n-2}$ for each positive integer $j$ reveals that $r^{j+1} / u^{j}$ is in $\Gamma^{2 n-2}$ for all $j$.

We can assume that

$$
u(z, x)=h(z, x)\left(z^{m}+\sum_{k=1}^{m-1} b_{k}(x) z^{k}\right)
$$

where $h(0,0) \neq 0$ and $h \in \Gamma^{2 n-2}$ and the $b_{k}$ 's are smooth. Let $v(z, x)$ $=z^{m}+\sum_{k=1}^{m-1} b_{k}(x) z^{k}$. At this point, we want to show that the derivatives 
$\left(\partial^{v} / \partial z^{v}\right)(r / v)(z, x)$ are uniformly bounded by a constant $C_{v}$ which is independent of $x$ for $x$ in a neighborhood of 0 . This is accomplished via

Lemma 2. Let $U_{R}=\{z \in U:|z| \leqslant R\}$ and let $r_{k}(z)$ and $v_{k}(z)$ be sequences of polynomials of the form

$$
r_{k}(z)=\sum_{j=0}^{m-1} a_{j, k^{z^{j}}} \text { and } v_{k}(z)=z^{m}+\sum_{j=0}^{m-1} b_{j, k^{z}}
$$

where the coefficients $a_{j, k}$ and $b_{j, k}$ are bounded in absolute value by a constant $M$ for all $j$ and $k \geqslant 0$. Assume also that for each positive integer $N$, there is a constant $C_{N}>0$ such that for all $k>0$,

$$
\operatorname{Sup}_{z \in U_{R}}\left|D^{N} \frac{r_{k}^{m+1}}{v_{k}^{m}}(z)\right| \leqslant C_{N}
$$

Then there are constants $C_{N}^{\prime}$ such that for all $k$,

$$
\operatorname{Sup}_{z \in U_{R / 2}}\left|D^{N} \frac{r_{k}}{v_{k}}(z)\right| \leqslant C_{N}^{\prime}
$$

Using the lemma, one immediately obtains that the derivatives of $f_{1}(z, x)$ in the $z$ variable are bounded in a neighborhood of $(0,0)$ in $U \times \mathbf{R}^{2 n-2}$. In the original coordinates, we have that the derivatives

$$
\left|\frac{\partial^{N}}{\partial z_{1}^{N}} f_{1}\left(z_{1}, z_{2}, \ldots, z_{n}\right)\right|
$$

are bounded near $z_{0}$ independent of $\left(z_{1}, \ldots, z_{n}\right)$.

Since there is a dense open subset of complex directions $z_{1}$ for which the above procedure can be carried out, it follows easily that $f_{1}$ is smooth up to the boundary near $z_{0}$. All the other components of $f$ are treated analogously.

Proof of Lemma 1. The classical Remmert proper mapping theorem states that $f$ is a branched cover of some finite order $m$. Let $F_{1}, F_{2}, \ldots, F_{m}$ denote the $m$ inverses to $f$ which can be defined locally on $D_{2}$ away from the image of the branch locus of $f$. In [3], it is shown that any symmetric function of $F_{1}, F_{2}, \ldots, F_{m}$ extends smoothly to $\bar{D}_{2}$. Hence, the $n$ functions defined on $\mathrm{C}^{n} \times \mathrm{D}_{2}$ given by

$$
P_{i}(z, w)=\prod_{k=1}^{m}\left(z_{i}-F_{k}(w)_{i}\right)
$$

are in $C^{\infty}\left(\mathbf{C}^{n} \times \bar{D}_{2}\right)$. Note that if $w=f(z)$, then $P_{i}(z, w)=0$ for $i=1,2, \ldots, n$.

Let $z_{0}$ be a boundary point of $D_{1}$ and let $B_{\delta}$ denote the ball of radius $\delta$ about $z_{0}$. Using the polynomials $P_{i}$ and the fact proved in Range [13] and 
Fornaess [9] that there is a positive integer $\eta$ such that

$$
\operatorname{dist}\left(z, b D_{1}\right)^{\eta} \leqslant \operatorname{dist}\left(f(z), b D_{2}\right) \leqslant \operatorname{dist}\left(z, b D_{1}\right)^{1 / \eta},
$$

it can be shown that the volume $B_{\delta} \cap D_{1}$ is mapped under $f$ onto a volume whose measure is greater than a constant $C$ times $\delta^{M}$ where $M$ and $C$ are independent of $\delta$. Hence, using the fact that $|u|^{2}$ is equal to the real Jacobian determinant of $f$, we obtain that

Therefore,

$$
\int_{B_{\delta} \cap D_{1}}|u|^{2} \geqslant C \delta^{M}
$$

$$
\operatorname{Sup}_{B_{\delta} \cap D_{1}}|u|^{2} \geqslant C^{\prime} \delta^{M^{\prime}}
$$

for some constants $C^{\prime}$ and $M^{\prime}$ which are independent of $\delta$. Hence $u$ vanishes to at most finite order at $z_{0}$.

REMARK. It is interesting to note that the assumption that the domains $D_{1}$ and $D_{2}$ are pseudoconvex is only used at one point in the proof of Theorem 1. Pseudoconvexity is only assumed in order to obtain the crucial inequality

$$
\operatorname{dist}\left(z, b D_{1}\right)^{\eta} \leqslant \operatorname{dist}\left(f(z), b D_{2}\right) \leqslant \operatorname{dist}\left(z, b D_{1}\right)^{1 / \eta} .
$$

\section{REFERENCES} 103-113.

1. S. Bell, Biholomorphic mappings and the $\bar{\partial}$-problem, Ann. of Math. (2) 114 (1981),

2. Analytic hypoellipticity of the $\bar{\partial}-$ Neumann problem and extendability of holomorphic mappings. Acta Math. 147 (1981), 109-116.

3. - Proper holomorphic mappings and the Bergman projection, Duke Math. J. 48 (1981), 167-175.

4. S. Bell and D. Catlin, Boundary regularity of proper holomorphic mappings, Duke Math. J. 49 (1982).

5. S. Bell and E. Ligocka, $A$ simplification and extension of Fefferman's theorem on biholomorphic mappings, Invent. Math. 57 (1980), 283-289.

6. K. Diederich and J. E. Fornaess, Pseudoconvex domains with real analytic boundary, Ann. of Math. (2) 107 (1978), 371-384.

7. C. Fefferman, The Bergman kernel and biholomorphic mappings of pseudoconvex domains, Invent. Math. 26 (1974), 1-65.

8. J. E. Fornaess, personal communication.

9. Biholomorphic mappings between weakly pseudoconvex domains, Pacific J. Math. 74 (1978), 63-65.

10. M. Golubitsky and V. Guillemin, Stable mappings and their singularities, SpringerVerlag, Heidelberg, 1973.

11. J. J. Kohn, Harmonic integrals on strongly pseudoconvex manifolds. I and II, Ann. of Math. (2) 78 (1963), 112-148; 79 (1964), 450-472.

12. Subellipticity of the sufficient conditions, Acta Math. 142 (1979), 79-122.

13. R. M. Range, The Carathéodory metric and holomorphic maps on a class of weakly pseudoconvex domains, Pacific J. Math. 78 (1978), 173-189.

DEPARTMENT OF MATHEMATICS, PRINCETON UNIVERSITY, PRINCETON, NEW JERSEY 08544 